\title{
Expression and distribution of Src in the nucleus of myocytes in cardiac hypertrophy
}

\author{
PING CHEN ${ }^{1}$, FAQIAN LI ${ }^{2}$, ZEQING XU ${ }^{1}$, ZHANYU LI $^{1}$ and XIAN PING YI ${ }^{1}$ \\ ${ }^{1}$ Department of Pathology, The Fifth Affiliated Hospital, Sun Yat-sen University, Zhuhai, Guangdong 519000, P.R. China; \\ ${ }^{2}$ Department of Pathology and Laboratory Medicine, University of Rochester Medical Center, Rochester, NY 14642, USA
}

Received March 3, 2013; Accepted May 3, 2013

DOI: $10.3892 /$ ijmm.2013.1382

\begin{abstract}
The Src kinase is involved in signaling events leading to cardiac hypertrophy. The exact effects of tyrosine phosphorylation and subnuclear distribution on cardiac hypertrophy and failure remain to be investigated. In this study, we examined the intranuclear expression and distribution of c-Src, Src phosphorylated at tyrosine 529 (Src[pY529]), Src phosphorylated at tyrosine 418 (Src[pY418]) and Src phosphorylated at tyrosine 215 (Src[pY215]) in the myocardial nuclei of the left ventricle (LV) from 2-, 6-, 12- and 18-month-old spontaneously hypertensive heart failure (SHHF) rats and age-matched Wistar-Kyoto (WKY) rats as normotensive controls by western blot analysis, immunofluorescent labeling and immunoprecipitation. Cellular $\mathrm{Src}$ (c-Src) expression in the myocardial nuclei of the LV of the 2-, 6-, 12- and 18-month-old SHHF rats was not significantly different from that in the myocardial nuclei of the LV of the age-matched WKY rats. Although there were no significant differences observed between the levels of Src[pY529] and Src[pY418] in the myocardial nuclei of the LV of the 2-monthold SHHF and WKY rats, the expression of Src[pY529] significantly decreased, while that of Src[pY418] significantly increased in the myocardial nuclei of the LV of the 6-, 12- and 18-month-old SHHF rats compared to the age-matched WKY controls. Furthermore, as demonstrated by double labeling with antibodies against fibrillarin and Src-associated in mitosis 68 kDa (Sam68), c-Src was co-localized with both Sam68 and fibrillarin in the nuclei; Src[pY529] co-localized with fibrillarin, but Src[pY418] co-localized with Sam68. The results from the present study suggest that the dephosphorylation of Src tyrosine kinase 529, the phosphorylation of tyrosine 418 and their subnuclear redistribution are involved in endonuclear signal transduction in cardiac myocytes, which regulates the
\end{abstract}

Correspondence to: Professor Xian Ping Yi, Department of Pathology, The Fifth Affiliated Hospital, Sun Yat-sen University, 52 Meihua East Road, Zhuhai, Guangdong 519000, P.R. China E-mail: yixianping83@yahoo.cn

Key words: cardiac hypertrophy, cell signaling, heart failure, hypertension, Src kinase development and progression of LV eccentric hypertrophy induced by hypertension.

\section{Introduction}

Hypertension is a factor responsible for structural and functional alterations in myocardial tissue leading to hypertrophy of the left ventricle (LV), which is defined as an increase in the LV mass in a compensatory response to elevated blood pressure to minimize wall stress (1). Eventually, congestive heart failure (CHF) develops after sustained load increase. LV hypertrophy is a strong independent predictor of cardiovascular events and all-cause mortality. Patients with LV hypertrophy are at an increased risk of stroke, coronary heart disease, $\mathrm{CHF}$ and sudden cardiac death, while the regression of LV hypertrophy is associated with the reduced risk of these clinical events $(2,3)$. Therefore, identifying the molecular mechanisms behind the mechanical stress from elevated blood pressure in LV hypertrophy is key to understanding and treating hypertensive heart disease. The Src kinase plays a critical role in the development of cardiac hypertrophy in response to mechanical stress (4).

The Src kinase, a non-receptor protein tyrosine kinase, participates in multiple signaling pathways involved in cardiac hypertrophy, such as integrin-mediated and $\mathrm{G}$ protein-coupled receptor signal transduction (5-10). Src kinase activity can be regulated by altering the phosphorylation of specific tyrosine residues. There are 3 main phosphorylation sites, tyrosines 529,418 and 215, that are involved in regulating Src activity. Phosphorylation at residues 418 and 215 increases Src kinase activity, while phosphorylation at tyrosine 529 downregulates Src kinase activity in growth factor signaling $(11,12)$. Src autophosphorylates, and Src kinase activity also transiently increases when cultured rat cardiac myocytes are subjected to mechanical stress in vitro (5). The Src kinase is also activated during cardiac hypertrophy following acute pressure overload in vivo (9). Our previous study demonstrated that the Src kinase relocated from the cytoplasm to the cell membrane and aggregated in the intercalated discs during decompensated remodeling of cardiac myocytes in LV hypertrophy resulting from hypertension (13). However, whether the Src kinase is involved in signal transduction in the nuclei of cardiac myocytes in the LV during hypertrophy resulting from hypertension remains unknown. 


\section{Materials and methods}

Animals. Two-, 6-, 12- and 18-month-old, lean, male spontaneously hypertensive heart failure (SHHF) rats and age-matched, male Wistar-Kyoto (WKY) rats, as the normotensive controls, were acquired from Da-Shuo Scientific Co. (Chengdu, China). Six rats from each age group were used for extracting nuclear protein (NP) homogenates of the LV tissues for western blot analysis and for cutting frozen sections of the LV tissues for immunofluorescent staining. Six rats from each age group were used for isolating cardiac myocytes for immunofluorescent staining. Six of the 6-month-old SHHF and WKY rats (6 rats from each) were used for immunoprecipitation. All procedures were performed in accordance with the Guide for the Care and Use of Laboratory Animals (US Department of Health and Human Services, NIH publication no. 85-23) and approved by the Sun Yat-sen University Animal Care and Use Committee.

Antibodies. Rabbit polyclonal antibodies against the $\mathrm{N}$-terminus of $\operatorname{Src}(\mathrm{N}-16)$ for western blot analyses, mouse monoclonal antibody against the N-terminus of $\mathrm{Src}(\mathrm{H}-12)$ for immunofluorescent labeling and immunoprecipitation, and mouse monoclonal antibody against Src-associated in mitosis $68 \mathrm{kDa}$ (Sam68) were obtained from Santa Cruz Biotechnology, Inc. (Santa Cruz, CA, USA). Anti-Src phosphorylated at tyrosine 529 (Src[pY529]) and anti-Src phosphorylated at tyrosine 418 ( $\mathrm{Src}[\mathrm{pY} 418])$ phosphospecific antibodies for western blot analysis and immunolabeling were obtained from Abcam (Cambridge, UK) and BioSource International, Inc. (Camarillo, CA, USA), respectively. Anti-Src phosphorylated at tyrosine 215 (Src[pY215]) phosphospecific antibody was obtained from Upstate Biotechnology, Inc. (Lake Placid, NY, USA). Anti-fibrillarin monoclonal antibody was obtained from Cytoskeleton, Inc. (Denver, CO, USA). Alexa Fluor 488 or 568 conjugated goat anti-rabbit $\operatorname{IgG}$ or goat anti-mouse IgG antibodies for immunolabeling were obtained from Molecular Probes (Eugene, OR, USA).

Myocyte isolation, frozen sections of intact myocardium, immunolabeling and confocal microscopy. Cardiac myocytes were enzymatically isolated as previously described (14). Briefly, the hearts were removed and trimmed. The aorta was cannulated for retrograde perfusion with oxygenated calciumfree Joklik medium (Sigma, St. Louis, MO, USA) at $37^{\circ} \mathrm{C}$ followed by incubation with the same medium containing $0.1 \%$ collagenase. After collagenase perfusion, the heart became softened and was removed from the perfusion apparatus. Tissue was minced in calcium-free Joklik medium and the isolated cells were filtered through a nylon mesh $(250 \mu \mathrm{m})$ into $8 \%$ paraformaldehyde solution, fixed at a final concentration of $4 \%$ for $10 \mathrm{~min}$ and subsequently suspended in phosphate-buffered saline (PBS) for fluorescent labeling.

The LV tissue was placed in embedding medium and frozen. Sections $(6-\mu \mathrm{m}$-thick) were cut using a cryostat and mounted onto poly-l-lysine-coated slides for immunofluorescent labeling. Frozen tissue sections were thawed to room temperature, fixed in cold acetone for $10 \mathrm{~min}$ and washed with PBS as previously described (15). For the isolated cardiac myocytes, cardiac myocyte suspensions were aliquoted onto positively charged slides, permeated with $0.5 \%$ Triton X-100 for $30 \mathrm{~min}$ at room temperature and washed in PBS (16). Immunofluorescent labeling was performed on both the frozen tissue sections and the isolated cardiac myocyte sedimentary slides according to the following procedure: After washing with PBS, the slides were blocked with $1 \%$ bovine serum albumin to reduce nonspecific binding and a primary antibody (anti-c-Src antibody or anti-Src[pY529], anti-Src[pY418] and anti-Src[pY215] phosphopecific antibodies, or anti-fibrillarin antibody or anti-Sam68 antibody) was added for overnight incubation at $4^{\circ} \mathrm{C}$. The primary antibody was removed and the slides were washed 3 times with PBS. A secondary fluorochrome-conjugated antibody (Alexa Fluor 488 conjugated goat anti-rabbit IgG antibody, Alexa Fluor 488 conjugated goat anti-mouse IgG antibody, Alexa Fluor 568 conjugated goat anti-rabbit IgG antibody or Alexa Fluor 568 conjugated goat anti-mouse IgG antibody, based on the primary antibody used in labeling) was added followed by incubation for $1 \mathrm{~h}$ at room temperature. Unbound secondary antibody was then washed away with PBS. The nuclei were counterstained with propidium iodide (PI). The same procedure was repeated with the second set of antibodies, except that the nuclei were not counterstained with PI for double labeling. The slides were mounted in 60\% glycerol in PBS and sealed with nail polish for observation using an Olympus FluoView Confocal Laser Scanning Microscope System. The negative controls were incubated with the omission or substitution of primary antibodies with rabbit serum under the same conditions. Cardiac myocytes labeled without primary antibody or with rabbit serum only showed weak diffuse background fluorescence.

$N P$ extraction, gel electrophoresis and western blot analysis. The extraction of NP was performed as previously described (17). NPs were separated by one-dimensional SDS-polyacrylamide gel electrophoresis (SDS-PAGE) (Laemmli) based on their molecular weight. NP content was determined for each sample using the Bradford method. Fifty micrograms of protein from each LV sample were mixed with Laemmli SDS sample buffer (2X stock Laemmli SDS sample buffer, 5\% 2-mercaptoethenol and $0.005 \%$ bromophenol blue), and the samples were then boiled and electrophoresed on $10 \%$ gels. The proteins were then transferred onto nitrocellulose membranes. The membranes were blocked with $3 \%$ BSA and 5\% dry milk in PBS for $1 \mathrm{~h}$ and probed with primary antibody. After thorough washing, the bound antibodies were visualized with horseradish peroxidase-conjugated anti-rabbit IgG antibody using the enhanced chemiluminescence technique (Amersham Biosciences, Piscataway NJ, USA). The protein bands were scanned and the densities were quantified using NIH image software. The mean optical density of each band was regarded as the density unit for the relative protein content.

Immunoprecipitation. The LV was homogenized with a PT1200C Polytron homogenizer in ice-cold lysis buffer $(50 \mathrm{mM}$ Tris. $\mathrm{HCl}, 1 \% \mathrm{NP}-40,150 \mathrm{mM} \mathrm{NaCl}, 1 \mathrm{mM}$ EDTA, $1 \mathrm{mM}$ PMSF, $1 \mu \mathrm{g} / \mathrm{ml}$ aprotinin, $1 \mu \mathrm{g} / \mathrm{ml}$ leupeptin, $1 \mu \mathrm{g} / \mathrm{ml}$ pepstatin, $1 \mathrm{mM} \mathrm{Na}_{3} \mathrm{VO}_{4}$ and $1 \mathrm{mM} \mathrm{NaF}, \mathrm{pH}$ 7.4). The homogenates were centrifuged at $14,000 \mathrm{x}$ g for $15 \mathrm{~min}$ at $4^{\circ} \mathrm{C}$. The supernatant was quantified using the Bradford method. A total of 1,000 $\mu \mathrm{g}$ protein was placed into a new centrifuge tube and adjusted to the same volume of $0.5 \mathrm{ml}$ with ice-cold lysis buffer. Following incubation with $30 \mu \mathrm{l}$ of $50 \%$ protein A agarose beads (Santa 
Cruz Biotechnology, Inc.) for $1 \mathrm{~h}$ at $4^{\circ} \mathrm{C}$ in a tube rotator, the beads were removed by centrifugation. Primary antibody was added to the supernatant and incubated overnight with rotation at $4^{\circ} \mathrm{C}$. The protein complex was pulled down by the addition of $30 \mu \mathrm{l}$ of $50 \%$ protein A agarose beads. Non-specific binding was removed by washing 3 times with ice-cold lysis buffer. The washed protein complex was boiled with $30 \mu \mathrm{l}$ of SDS sample buffer and separated by SDS-PAGE for western blot analysis. For the negative controls, irrelevant antibody or rabbit serum was used instead of specific antibodies.

Statistical analysis. Data are expressed as the means $\pm \mathrm{SE}$. Two-sample, independent-group t-tests were performed to compare physiological data and the optical densities from the western blot analyses between the SHHF and WKY rats. A value of $\mathrm{P}<0.05$ was considered to indicate a statistically significant difference.

\section{Results}

Comparison of physiological parameters between SHHF and WKY rats. The heart weight ( $\mathrm{HW})$, the ratio of the heart weight to the body weight $(\mathrm{HW} / \mathrm{BW})$ and the $\mathrm{LV}$ weight (LVW) significantly increased in the 2-, 6-, 12- and 18-month-old SHHF rats as compared with the age-matched WKY rats (Table I). These results confirm that heart hypertrophy, particularly LV hypertrophy, exists in 2-, 6-, 12- and 18-month-old SHHF rats.

Nuclear expression of c-Src, Src[pY529], Src[pY418] and Src [pY215] in the LV of SHHF rats. We used rabbit antibodies against the N-terminus of cellular Src (c-Src) to detect its expression in the NP extracts from the LV of SHHF and WKY rats. Western blot analysis revealed the expected band at $60 \mathrm{kDa}$, which represents $\mathrm{c}-\mathrm{Src}$. There were no significant differences observed in c-Src expression in the NP between the 2-, 6-, 12- and 18-month-old SHHF rats and their agematched WKY controls $(104.5 \pm 11.1$ vs. $104.4 \pm 10.7,105.2 \pm 9.5$ vs. $104.5 \pm 9.2,113.9 \pm 10.9$ vs. $104.1 \pm 12.7$ and $126.9 \pm 10.3$ vs 111.7 \pm 10.7 , respectively; $\mathrm{N}=6, \mathrm{P}>0.05$ ) (Fig. 1A).

The activation of Src kinases is closely associated with the phosphorylation and dephosphorylation of certain amino sites. To determine whether Src phosphorylation levels were altered in the NP extracts from the LV of the SHHF rats, we investigated 3 tyrosine phosphorylation sites: Src[pY529], Src[pY418] and Src[pY215]. Western blot analysis revealed that the Src[pY529] levels in the NP extracts from the LV did not significantly differ between the 2-month-old SHHF rats and the age-matched WKY control rats $(126.5 \pm 5.0$ vs. $122.2 \pm 5.0$; $\mathrm{N}=6, \mathrm{P}>0.05$ ) (Fig. 1B). However, Src[pY529] levels decreased in the NP extracts from the LV tissues from the 6-, 12- and 18-month-old SHHF rats compared to the age-matched WKY rats $(101.6 \pm 8.7$ vs. $128.8 \pm 6.2,104.6 \pm 7.1$ vs. $128.5 \pm 5.0$ and $102.0 \pm 8.9$ vs. $132.1 \pm 7.1$, respectively; $\mathrm{N}=6, \mathrm{P}<0.05$ ) (Fig. $1 \mathrm{~B}$ ). Src phosphorylation levels at the tyrosine 529 site were downregulated in the nuclei of the cardiac myocytes of the hypertrophic LV of the 6- to 18-month-old SHHF rats.

Src[pY418] expression in the NP extracts from the LV myocytes of the 2-month-old SHHF rats did not differ significantly from that of the 2-month-old WKY rats $(47.0 \pm 5.6$ vs.
Table I. Comparison of the physiological data.

\begin{tabular}{llll}
\hline Group & HW, & HW/BW, g & LVW, g \\
\hline $2 \mathrm{M}$, SHHF & $0.85 \pm 0.03^{\mathrm{a}}$ & $0.40 \pm 0.01^{\mathrm{a}}$ & $0.51 \pm 0.02^{\mathrm{a}}$ \\
$2 \mathrm{M}, \mathrm{WKY}$ & $0.62 \pm 0.01$ & $0.33 \pm 0.01$ & $0.31 \pm 0.01$ \\
$6 \mathrm{M}, \mathrm{SHHF}$ & $1.18 \pm 0.05^{\mathrm{a}}$ & $0.40 \pm 0.01^{\mathrm{a}}$ & $0.70 \pm 0.03^{\mathrm{a}}$ \\
$6 \mathrm{M}, \mathrm{WKY}$ & $0.99 \pm 0.02$ & $0.30 \pm 0.00$ & $0.52 \pm 0.01$ \\
$12 \mathrm{M}, \mathrm{SHHF}$ & $1.35 \pm 0.02^{\mathrm{a}}$ & $0.43 \pm 0.00^{\mathrm{a}}$ & $0.78 \pm 0.02^{\mathrm{a}}$ \\
$12 \mathrm{M}, \mathrm{WKY}$ & $1.20 \pm 0.03$ & $0.26 \pm 0.00$ & $0.64 \pm 0.01$ \\
$18 \mathrm{M}, \mathrm{SHHF}$ & $1.59 \pm 0.03^{\mathrm{a}}$ & $0.42 \pm 0.01^{\mathrm{a}}$ & $1.01 \pm 0.03^{\mathrm{a}}$ \\
$18 \mathrm{M}, \mathrm{WKY}$ & $1.40 \pm 0.04$ & $0.25 \pm 0.00$ & $0.79 \pm 0.02$
\end{tabular}

Values are the means \pm SEM, age in months (M); HW, heart weight; $\mathrm{HW} / \mathrm{BW}$, ratio of heart weight to body weight; LVW, left ventricular weight; SHHF, spontaneously hypertensive heart failure; WKY, Wistar-Kyoto rats; ${ }^{\mathrm{a}} \mathrm{P}<0.05$ compared with age-matched WKY control rats.

49.3 $\pm 5.0 ; \mathrm{N}=6, \mathrm{P}>0.05$ ) (Fig. 1C). However, Src[pY418] expression significantly increased in the NP extracts from the LV myocytes from the 6-, 12- and 18-month-old SHHF rats compared to the age-matched WKY rats $(71.2 \pm 5.1$ vs. $54.8 \pm 5.6,72.3 \pm 6.3$ vs. $54.3 \pm 3.9$ and $82.9 \pm 5.8$ vs. $63.1 \pm 6.2$, respectively; $\mathrm{N}=6, \mathrm{P}<0.05$ ) (Fig. $1 \mathrm{C}$ ). Src phosphorylation levels at the tyrosine 418 site increased in the nuclei of cardiac myocytes from the hypertrophic LV of the 6- to 18-month-old SHHF rats.

Finally, Src[pY215] levels were not significantly altered in the NP extracts from the LV tissues from the 2-, 6-, 12and 18-month-old SHHF rats and the age-matched WKY control rats $(101.6 \pm 7.8$ vs. $114.9 \pm 10.8,110.3 \pm 5.4$ vs. $114.4 \pm 6.0$, $105.6 \pm 4.9$ vs. $100.1 \pm 8.0$ and $108.6 \pm 8.9$ vs. $114.0 \pm 7.4$, respectively; $\mathrm{N}=6, \mathrm{P}>0.05$ ) (Fig. 1D).

Subnuclear localization of $c$-Src in the nuclei of cardiac myocytes. The subcellular localization of the Src kinase is important for the regulation of specific cellular processes, such as mitogenesis, cytoskeletal organization and membrane trafficking (18). To further investigate the subnuclear distribution and localization of c-Src in the nuclei of cardiac myocytes, we labeled the cardiac myocytes from the LV of the 2-, 6-, 12- and 18-month-old SHHF rats and the age-matched WKY rats with anti-c-Src antibody, which we detected using immunofluorescence. The result was that fluorescent brightness was uniformly distributed in the nuclei of the cardiac myocytes from all the rats (data not shown). When the slides were scanned using higher magnification and lower photomultiplier tube voltage and gain settings on the confocal microscope to decrease fluorescent background brightness, we clearly observed bright fluorescent dots of $\mathrm{c}$-Src in the nuclei of the cardiac myocytes. Subsequent double labeling of the cardiac myocyte sections with anti-c-Src antibody and anti-Sam68 or anti-fibrillarin antibody revealed that c-Src was co-localized mainly with Sam68 (Fig. 2A-C). A smaller fraction of c-Src co-localized with fibrillarin (Fig. 2D-F) in the nuclei of the cardiac myocytes. 
A

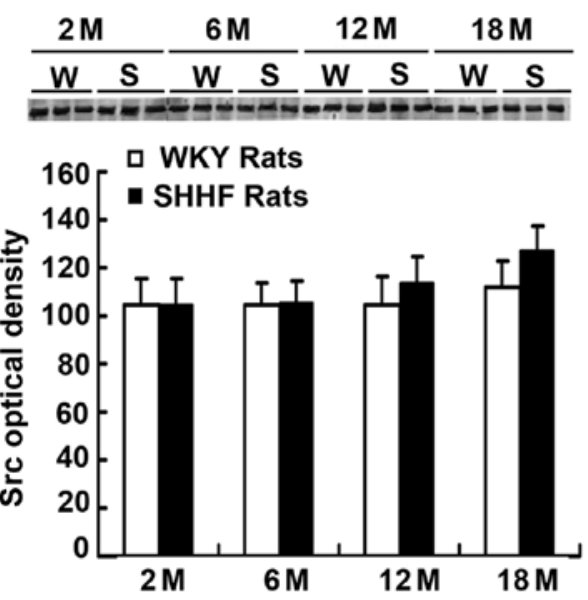

C
B
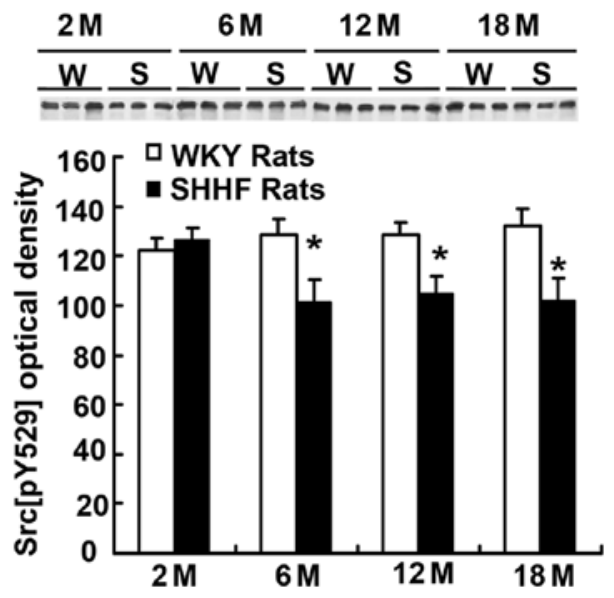

D

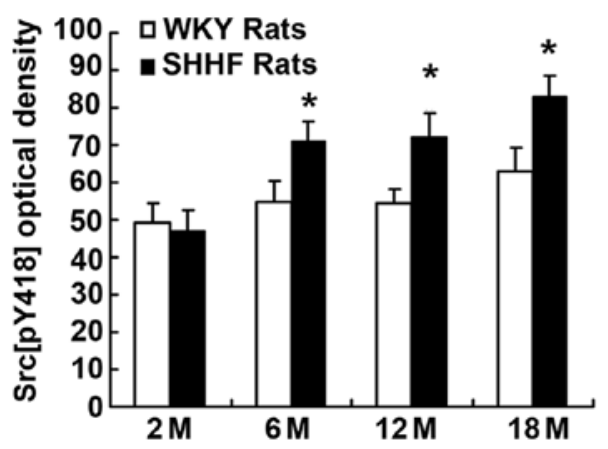

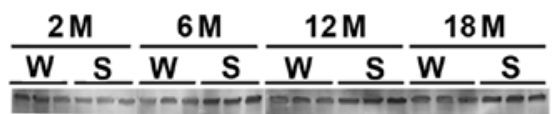

*
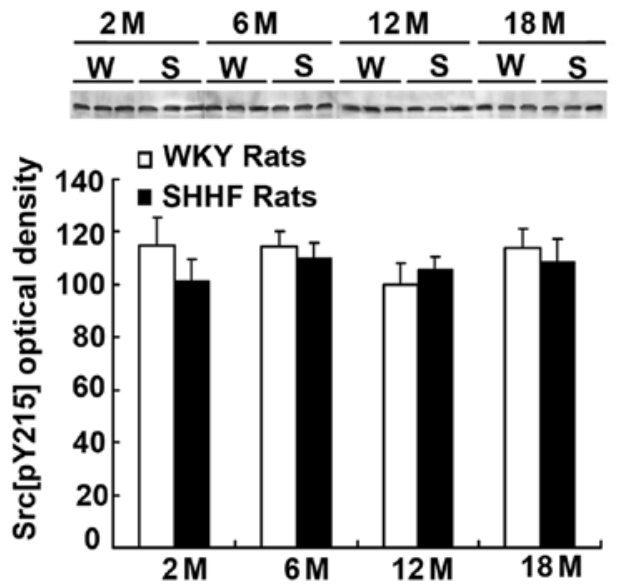

Figure 1. (A) c-Src, (B) Src[pY529], (C) Src[pY418] and (D) Src[pY215] expression in the nuclear protein (NP) fraction of the left ventricle of 2- (2 M), 6- (6 M), 12- (12 M), 18- (18 M) month-old spontaneously hypertensive heart failure (SHHF) (S) and [Wistar-Kyoto (WKY)] (W) rats (N=6). (A) Western blot (top panel) showing a similar expression pattern of c-Src between the SHHF and WKY rats; histogram (bottom panel) also demonstrates that there is no significant difference in c-Src content between the SHHF and WKY rats by densitometry analysis ( $\mathrm{N}=6, \mathrm{P}>0.05$ ). (B) Western blot (top panel) and histrogram (bottom) showing a similar level of Src[pY529] between the 2-month-old SHHF and WKY rats by densitometry analysis; the level of Src[pY529] in the 6-, 12- and 18-month-old SHHF rats is significantly lower than that in the age-matched WKY rats $(\mathrm{N}=6, \mathrm{P}<0.05)$. (C) Western blot (top panel) and histogram (bottom panel) also demonstrate a similar level of Src[pY418] between the 2-month-old SHHF and WKY rats by densitometry analysis; the level of Src[pY418] in the 6-, 12- and 18-month-old SHHF is significantly higher than that of the age-matched WKY rats ( $\mathrm{N}=6, \mathrm{P}<0.05)$. (D) Western blot (top panel) and histogram (bottom panel) show a similar level of Src[pY215] between the 2-, 6-, 12- and 18-month-old SHHF and age-matched WKY rats by densitometry analysis (N=6, P $>0.05)$. ${ }^{*} \mathrm{P}<0.05$ compared with the age-matched WKY control rats. $\mathrm{N}$, number of animals.

Immunoprecipitation also showed that c-Src co-localized with Sam68 and fibrillarin in the nuclei of the cardiac myocytes and that the amount of c-Src associated with Sam68 and fibrillarin increased in the cardiac myocytes of the hypertrophic LV in the 6-month-old SHHF rats compared to the age-matched WKY controls (Fig. 2G).

Subnuclear localization of Src[pY529] in the nuclei of cardiac myocytes. To investigate the distribution and localization of Src[pY529] in the nuclei of cardiac myocytes of the hypertrophic LV of the SHHF rats, we performed immunofluorescent labeling of Src[pY529] on the cardiac myocytes that were isolated from the LV of the 2-, 6-, 12- and 18-month-old SHHF rats and the age-matched WKY controls. The results revealed that a similar pattern of $\operatorname{Src}[\mathrm{pY} 529]$ fluorescence was distributed in the nuclei of the cardiac myocytes from the 6 , 12 and 18-month-old SHHF rats; Src[pY529] staining revealed
1-4 bright fluorescent dots on a diffusely weak fluorescent background in the nuclei of the cardiac myocytes (Fig. 3A-F). It was further demonstrated, by means of the double staining of Src[pY529] with antibodies against fibrillarin and Sam68, that the bright green dot-like fluorescence of Src[pY529] overlaid with the red dot-like fluorescence of fibrillarin; thus, Src[pY529] co-localized with fibrillarin in the nucleoli, but not with Sam68 (Fig. 3G-L). In addition, it was also shown, in the immunoprecipitation with anti-Src[pY529] antibody followed by western blot analysis with anti-fibrillarin antibody, that Src[pY529] co-localized with fibrillarin; this was more evident in the cardiac myocytes of the hypertrophic LV from the 6-month-old SHHF rats compared to the age-matched WKY controls (Fig. 3M).

Subnuclear localization of Src[pY418] in the nuclei of cardiac myocytes. To investigate the distribution and localization 



Figure 2. (A-F) Double labeling of c-Src (green, A and D) with Sam68 (red, B) or fibrillarin (red, E) in cardiac myocytes isolated from the left ventricle of 6-month-old spontaneously hypertensive heart failure (SHHF) rats. (C and F) The merged images are shown in the right column. (A-C) The bright green fluorescent dots of c-Src are co-localized with the red fluorescence of Sam68, and (D-F) a small portion of the c-Src fluorescent dots overlap with the red fluorescent fibrillarin dot in the nuclei of the cardiac myocytes. Scale bar, $10 \mu \mathrm{m}$. (G) Immunoprecipitation (IP) with anti-c-Src antibody revealed similar amounts of c-Src in the cardiac myocytes of the hypertrophic left ventricle from the 6-month-old SHHF rats and the age-matched Wistar-Kyoto (WKY) controls. Western blot (WB) with anti-Sam68 or anti-fibrillarin antibody, showing that c-Src bound to Sam68 or fibrillarin increased significantly in the cardiac myocytes of the hypertrophic left ventricle from the 6-month-old SHHF rats compared to the age-matched WKY controls $(\mathrm{N}=6, \mathrm{P}<0.05)$.

of Src[pY418] in the nuclei of the cardiac myocytes of the hypertrophic LV from the SHHF rats, we labeled intact frozen sections of LV tissues from the 2-, 6-, 12- and 18-month-old SHHF rats and the age-matched WKY controls with antiSrc[pY418] antibody. Confocal microscopy revealed a similar pattern of macular-like fluorescent distribution of Src[pY418] in the nuclei of the cardiac myocytes from the 6- to 18-monthold SHHF rats (Fig. 4A-F). Double labeling demonstrated that Src[pY418] fluorescence was co-localized mainly with Sam68 but not with fibrillarin in the nuclei of the cardiac myocytes (Fig. 4G-L). Immunoprecipitation with anti-Sam68 antibody followed by western blot analysis with anti-Src[pY418] antibody confirmed that Src[pY418] co-localized with Sam68. The same technique also revealed that the expression of Sam68bound Src[pY418] increased in the cardiac myocytes of the hypertrophic LV from the 6-month-old SHHF rats compared to the age-matched WKY controls (Fig. 4M).

We also immunolabeled Src[pY215] on frozen sections of the LV from the 2-, 6-, 12- and 18-month-old SHHF rats and the age-matched WKY controls. No difference was observed between the Src[pY215] signals in the myocardial nuclei of the LV of the WKY and SHHF rats (data not shown).

\section{Discussion}

Hypertension-induced increases in sustained pressure load cause increased left ventricular wall stress and thereby stimulate myocardial hypertrophy (3). Wall stress stimulates sarcomeres to grow in a parallel pattern by increasing protein synthesis, which increases myocyte width in response to increased afterload (19). Cardiac hypertrophy, therefore, is a compensatory response to increased wall stress. However, with the progression of cardiac hypertrophy, the LV can no longer compensate for the sustained increased afterload and eccentric hypertrophy of the LV occurs, which eventually results in $\mathrm{CHF}(2,20)$.

In the SHHF rats, myocardial sarcomeres begin increasing in width at as early as 2 months and reach a maximum cross-sectional area at approximately 4-6 months (21). The pathological changes during this period include concentric left ventricular hypertrophy and the relative thickening of the LV wall. At 6 months, sarcomeres begin to increase in length, which results in progressive lengthening and maladaptive remodeling, leading to $\mathrm{CHF}$ at $18-24$ months $(21,22)$. The molecular mechanisms controlling 'a change-over switch' in the hypertrophic 

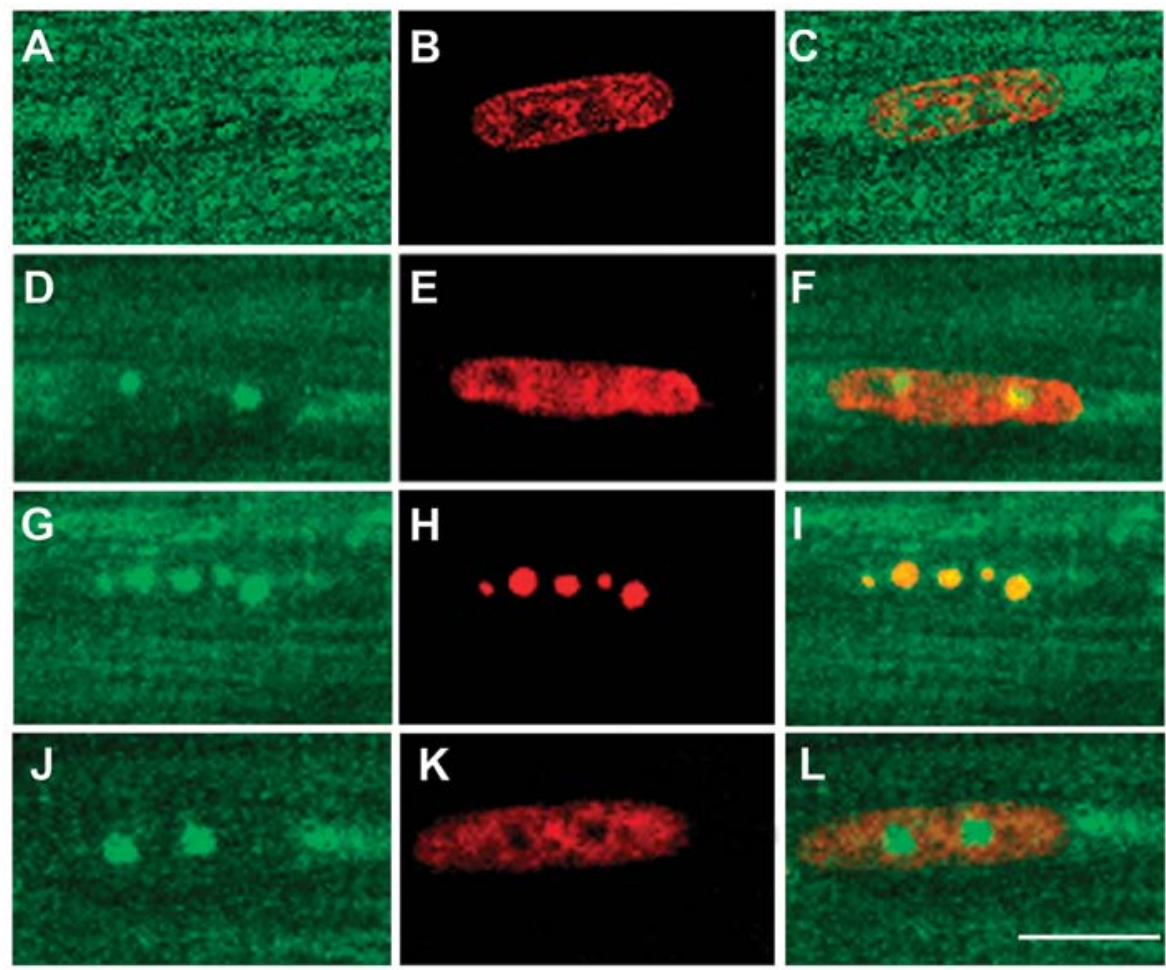

M

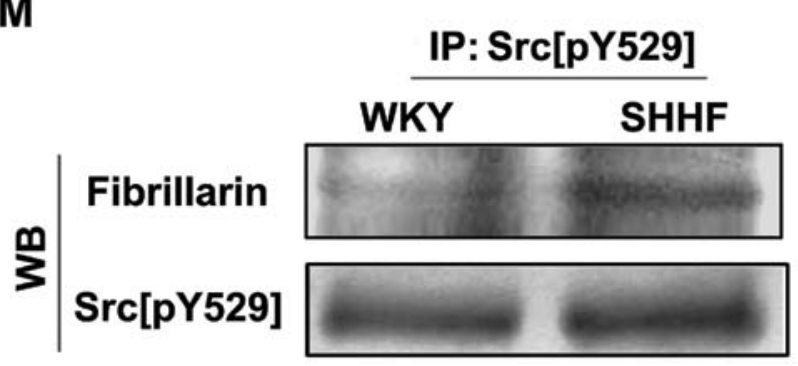

Figure 3. (A-F) Confocal microscopy images of (A) the evenly distributed green fluorescence of Src[pY529] and the myocardial nuclei [(B) red, counterstained with propidium iodide] of (A-C) 6-month-old Wistar-Kyoto (WKY) rats and (D) of 2 distinct bright green fluorescent dots against a relatively weakened fluorescence background of Src[pY529] and the myocardial nuclei [(E) red, counterstained with propidium iodide] of (D-F) 6-month-old spontaneously hypertensive heart failure (SHHF) rats. (C and F) Merged images are shown in the right column. (G-L) Double labeling of Src[pY529] (G and J, green) with fibrillarin (H, red) or Sam68 (K, red) shows that the Src[pY529] green fluorescence co-localized with the red fluorescence of fibrillarin, resulting in bright yellow fluorescent dots in the merged image (I). There is no overlap with the red fluorescence of Sam68 in the merged image (L) in the nuclei of the cardiac myocytes of the LV from the 6-month-old SHHF rats. Scale bar, $10 \mu \mathrm{m}$. (M) Immunoprecipitation (IP) with anti-Src[pY529] antibody followed by western blot analysis (WB) with antifibrillarin antibody. Levels of fibrillarin-bound Src[pY529] increased in the cardiac myocytes of the left ventricle of the 6-month-old SHHF rats compared to the age-matched WKY rats, whereas a similar level of Src[pY529] was observed by WB on the same membrane after stripping $(\mathrm{N}=6, \mathrm{P}<0.05)$.

pattern from cross-sectional growth to myocyte lengthening are not entirely clear. In the present study, we found that the late decompensatory eccentric hypertrophy of the LV occurred at 6-18 months. At the same time, the expression of Src[pY529] decreased and that of Src[pY418] increased in the nuclei of the myocytes in the hypertrophic LV from the SHHF rats. These results suggest that the dephosphorylation of Src tyrosine 529 and the phosphorylation of tyrosine 418 are involved in the regulation of cardiac remodeling in the development and progression of the decompensated hypertensive ventricular hypertrophy by the intranuclear signaling transduction pathway in cardiac myocytes.

From the N- to C-terminus, c-Src contains an SH4 domain, a unique domain, an SH3 domain that directs specific association with proline-rich motifs, an $\mathrm{SH} 2$ domain that provides interaction with phosphotyrosine motifs, an SH2-kinase linker, an SH1 domain that is responsible for the enzymatic activity and contains tyrosine 418 and a C-terminal regulatory segment that contains tyrosine 529 (23). Under basal conditions in vivo, the majority of Src is phosphorylated at tyrosine 529; phosphorylated tyrosine 529 binds intra-molecularly with the c-Src SH2 domain while the SH3 domain binds to prolinerich sequences located within the SH2-kinase linker. These 2 intramolecular associations stabilize a restrained form of the enzyme (23). Dephosphorylation of the negative regulatory site, tyrosine 529, is a mechanism by which Src may be activated in response to extracellular stimuli. Candidate phosphotyrosine 529 phosphatases include cytoplasmic protein-tyrosine phosphatase 1B (PTP1B) and transmembrane enzymes, such as CD45, PTP $\alpha$, PTPE and PTP $\lambda$ (12). An 

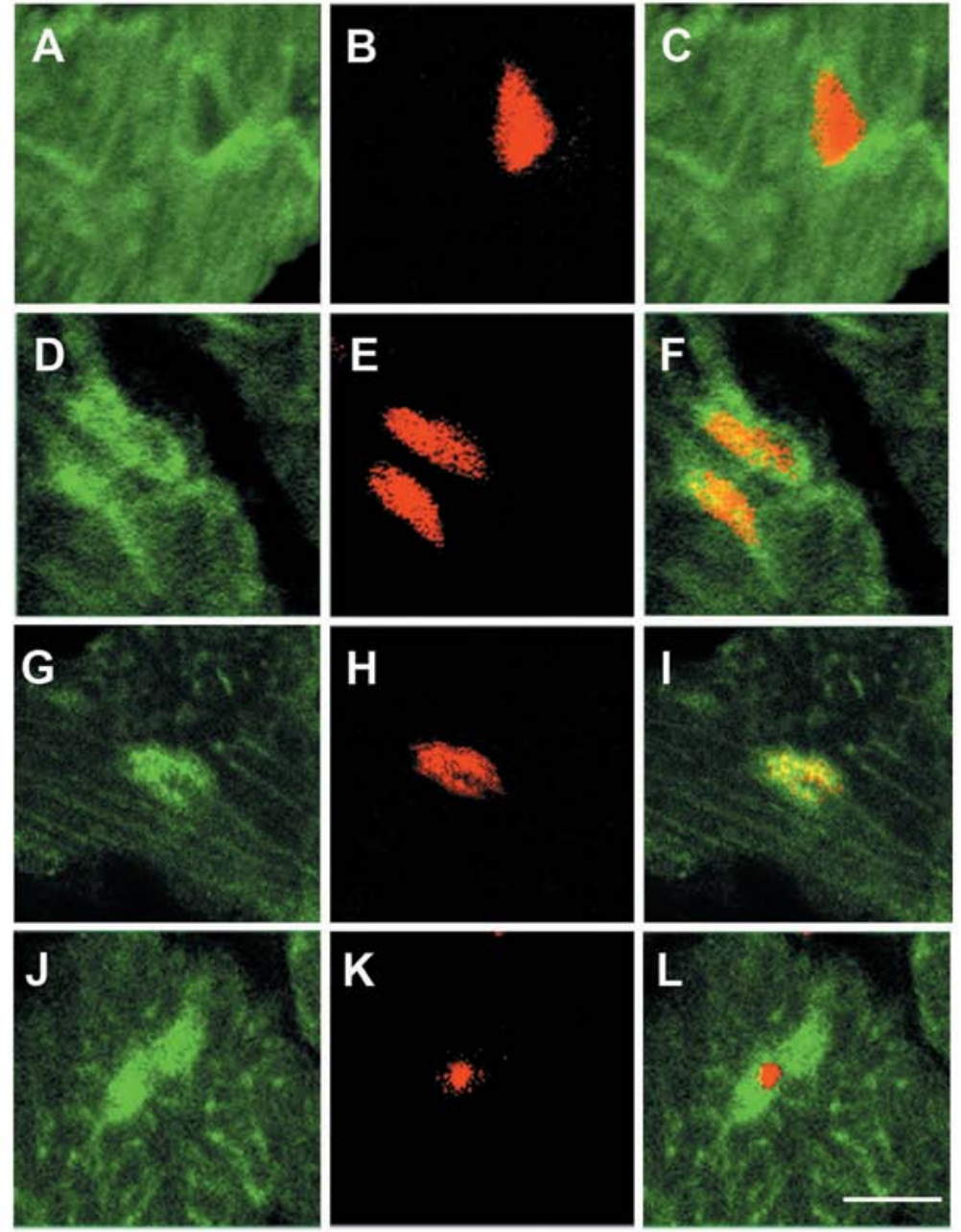

M

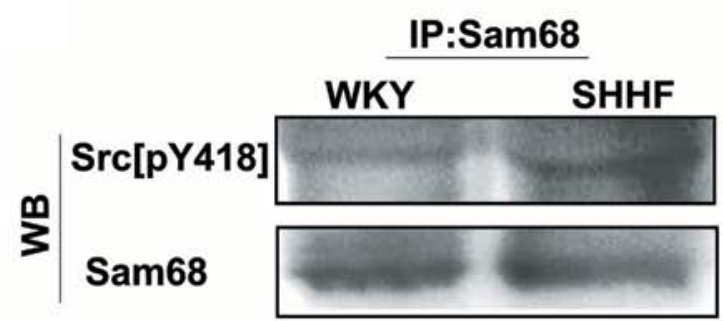

Figure 4. (A-F) Immunofluorescent labeling of Src[pY418] (A and D, green) and the myocardial nuclei [(B and E) red, counterstained with propidium iodide] in frozen sections of the LV tissues from (A-C) 6-month-old Wistar-Kyoto (WKY) rats and (D-F) spontaneously hypertensive heart failure (SHHF) rats. (A and C) Only a weak green Src[pY418] fluorescence is present in the myocardial nuclei of WKY rats, (D and F) but stronger green Src[pY418] fluorescence accumulated in the myocardial nuclei of the SHHF rats. (G-L) Double labeling of Src[pY418] (G and J, green) with Sam68 (H, red) or fibrillarin (K, red) shows that green fluorescent Src[pY418] co-localized with the red fluorescence of Sam68, which produced bright yellow fluorescence in the merged image (I). There was no overlap with the red fluorescent fibrillarin in the merged image (L) of the nuclei of the cardiac myocytes in frozen sections of the LV tissues from the 6-month-old SHHF rats. Scale bar, $10 \mu \mathrm{m}$. (M) Immunoprecipitation (IP) with anti-Sam68 antibody followed by western blot analysis (WB) with antiSrc[pY418] antibody. Levels of Src[pY418] bound to Sam68 increased in the cardiac myocytes of the left ventricle from the 6-month-old SHHF rats compared to the age-matched WKY controls, whereas a similar levels of Sam68 was observed down, as shown by WB on the same membrane after stripping $(\mathrm{N}=6, \mathrm{P}<0.05)$.

alternative mechanism of activating $\mathrm{Src}$ is the displacement of the negative regulatory element from the $\mathrm{SH} 2$ domain and/ or disruption of interaction by presenting higher-affinity $\mathrm{SH} 2$ or SH3 domain-binding ligands (24). In addition, Src with phosphorylated tyrosine 529 cannot undergo autophosphorylation at other sites, such as tyrosine 418; thus, the tyrosine 529 residue must first be dephosphorylated to allow Src autophosphorylation at other sites and activation (11). In the present study, we found that the expression of Src phosphorylated at tyrosine 529 decreased and that of Src phosphorylated at tyrosine 418 increased; Src[pY529] aggregated inhe nucleoli where it co-localized with a nucleolar protein, fibrillarin, in the nuclei of the cardiac myocytes of the hypertrophic LV from the SHHF rats. Src[pY529] aggregating in the nucleoli and binding fibrillarin may lead to the dissociation of pY529 from SH2, thereby disrupting the pY529-mediated auto-inhibition, as fibrillarin competitively occupies the $\mathrm{SH} 2$ domain. Enzymes such as PTP $\alpha$ may be involved in dephosphorylating 
pY529 from SH2. When pY529 dissociates from SH2, tyrosine 418, which is located in the SH1 domain, will be exposed, allowing it to undergo autophosphorylation, resulting in the increased expression of Src[pY418] and the activation of the tyrosine 418-phosphorylated Src kinase.

The nucleolus is the most active and dynamic nuclear domain that plays a prominent role in the organization of various components of the nucleus; it is not only essential for ribosomal production, but also for the control of cell survival and proliferation $(25,26)$. Fibrillarin is an evolutionarily conserved, obligatory protein component of eukaryotic cell nucleoli involved in the early processing and modification of pre-rRNA and ribosomal and nucleolar assembly; it is also essential for early embryonic development $(25,27)$. Moreover, fibrillarin depletion results in the reduction of cellular growth and abnormal nuclear morphology, suggesting that fibrillarin plays a critical role in cellular growth and the maintenance of nuclear shape (25). It is well known that cardiac myocytes are terminally differentiated cells with limited or no potential to re-enter the cell cycle (no-dividing cells), and therefore, the post-natal growth of cardiac myocytes occurs through hypertrophy of existing cells. The aggregation of Src[pY529] to the nucleoli and its co-localization with fibrillarin during hypertensive cardiac hypertrophy suggests that the Src kinase is involved in the hypertrophic growth of myocardial cells and plays a role in the endonucleolar signal transduction in myocardial hypertrophy that is induced by hypertension.

Sam68 is the prototypical member of the signal transducer and activator of RNA (STAR) family of RNA-binding proteins that link signaling pathways to RNA processing (28). Sam68 was first identified as the major phosphorylation substrate of the Src kinase during mitosis. It can not only interact with the SH2/SH3 domain-containing adaptor proteins and signaling enzymes, such as the Src family kinases, phosphatidylinositol 3-kinase (PI3K) and growth factor receptor-bound protein 2 (Grb2), but is also involved in several steps of mRNA processing, including transcription, alternative splicing and nuclear export $(29,30)$. Therefore, Sam68 functions as a multifunctional adaptor by linking signaling pathways to the transcriptional and post-transcriptional regulation of gene expression $(28,31)$. The co-localization of Src and Src[pY418] with Sam68, as observed in this study, suggests that the Src kinase regulates signal transduction in myocardial hypertrophy by directly controlling hypertrophic gene transcription in the nucleus. An earlier study demonstrated that SrcF527, a constitutively active oncogenic mutant of c-Src, stimulated hypertrophic expression in genes, such as atrial natriuretic factor (ANF), skeletal muscle (SkM)- $\alpha$-actin and $\beta$-myosin heavy chain ( $\beta$-MHC) (32). Thus, the Src kinase is a potential candidate for hypertrophic gene transcription and RNA processing in the nucleus during cardiac hypertrophy in chronic hypertension.

In conclusion, the data from the present study suggest that the Src kinase is involved in regulating cardiac myocyte remodeling in eccentric hypertrophy of the LV by participating in an intranuclear signaling transduction pathway, constituted by the dephosphorylation of Src tyrosine kinase 529, the phosphorylation of tyrosine 418, and intranuclear redistribution in the nuclei of myocardial cells undergoing hypertensive cardiac hypertrophy.

\section{Acknowledgements}

This study was supported by grants from the National Nature Science Foundation of China (no. 30871046) and the Nature Science Foundation of Guangdong Province, China (no. 8151008901000162).

\section{References}

1. Drazner MH: The progression of hypertensive heart disease. Circulation 123: 327-334, 2011.

2. Diamond JA and Phillips RA: Hypertensive heart disease. Hypertens Res 28: 191-202, 2005.

3. Krauser DG and Devereux RB: Ventricular hypertrophy and hypertension: prognostic elements and implications for management. Herz 31: 305-316, 2006.

4. Marin TM, Clemente CF, Santos AM, et al: Shp2 negatively regulates growth in cardiomyocytes by controlling focal adhesion kinase/Src and mTOR pathways. Circ Res 103: 813-824, 2008.

5. Torsoni AS, Constancio SS, Nadruz W Jr, Hanks SK and Franchini KG: Focal adhesion kinase is activated and mediates the early hypertrophic response to stretch in cardiac myocytes. Circ Res 93: 140-147, 2003.

6. Kuppuswamy D, Kerr C, Narishige T, Kasi VS, Menick DR and Cooper G IV: Association of tyrosine-phosphorylated c-Src with the cytoskeleton of hypertrophying myocardium. J Biol Chem 272: 4500-4508, 1997.

7. Kovacic B, Ilic D, Damsky CH and Gardner DG: c-Src activation plays a role in endothelin-dependent hypertrophy of the cardiac myocyte. J Biol Chem 273: 35185-35193, 1998.

8. Zou Y, Komuro I, Yamazaki T, et al: Both Gs and Gi proteins are critically involved in isoproterenol-induced cardiomyocyte hypertrophy. J Biol Chem 274: 9760-9770, 1999.

9. Heidkamp MC, Bayer AL, Scully BT, Eble DM and Samarel AM: Activation of focal adhesion kinase by protein kinase $\mathrm{C}$ epsilon in neonatal rat ventricular myocytes. Am J Physiol Heart Circ Physiol 285: H1684-H1696, 2003.

10. Aikawa R, Nagai $\mathrm{T}$, Kudoh $\mathrm{S}$, et al: Integrins play a critical role in mechanical stress-induced p38 MAPK activation. Hypertension 39: 233-238, 2002.

11. Roskoski R Jr: Src protein-tyrosine kinase structure and regulation. Biochem Biophys Res Commun 324: 1155-1164, 2004.

12. Roskoski R Jr: Src kinase regulation by phosphorylation and dephosphorylation. Biochem Biophys Res Commun 331: 1-14, 2005.

13. Li LH, Wang XH, Xie YY, et al: Membrane translocation of Src kinase in cardiac myocytes of hypertrophic left ventricle of hypertensive rats. Chinese Heart Journal 23: 295-299, 2011 (In Chinese).

14. Li ZY, Yi XP, Zhong L, et al: Expression of focal adhesion kinase in cardiac myocytes of hypertrophic ventricle. Zhonghua Bing Li Xue Za Zhi 36: 677-680, 2007 (In Chinese).

15. Zhong L, Yi XP, Li ZY and Faqian L: Phosphorylation and nuclear translocation of serine 722 and serine 910 of focal adhesion kinase in hypertrophic cardiac myocytes of left ventricle of spontaneously hypertensive rats. Zhonghua Bing Li Xue Za Zhi 37: 328-332, 2008 (In Chinese).

16. Yi XP, Gerdes AM and Li F: Myocyte redistribution of GRK2 and GRK5 in hypertensive, heart-failure-prone rats. Hypertension 39: 1058-1063, 2002.

17. Blough E, Dineen B and Esser K: Extraction of nuclear proteins from striated muscle tissue. Biotechniques 26: 202-204, 206, 1999.

18. Vielreicher M, Harms G, Butt E, Walter U and Obergfell A: Dynamic interaction between Src and C-terminal Src kinase in integrin alphaIIbbeta3-mediated signaling to the cytoskeleton. J Biol Chem 282: 33623-33631, 2007.

19. Papadopoulos DP and Papademetriou V: Hypertrophic and hypertensive hypertrophic cardiomyopathy - a true association? Angiology 61: 92-99, 2010.

20. Li F, Wang X, Yi XP and Gerdes AM: Structural basis of ventricular remodeling: role of the myocyte. Curr Heart Fail Rep 1: 5-8, 2004.

21. Heyen JR, Blasi ER, Nikula K, et al: Structural, functional, and molecular characterization of the SHHF model of heart failure. Am J Physiol Heart Circ Physiol 283: H1775-H1784, 2002. 
22. Johnsen DD, Kacimi R, Anderson BE, Thomas TA, Said S and Gerdes AM: Protein kinase $\mathrm{C}$ isozymes in hypertension and hypertrophy: insight from SHHF rat hearts. Mol Cell Biochem 270: 63-69, 2005.

23. Ingley E: Src family kinases: regulation of their activities, levels and identification of new pathways. Biochim Biophys Acta 1784 $56-65,2008$.

24. Schaller MD, Hildebrand JD and Parsons JT: Complex formation with focal adhesion kinase: A mechanism to regulate activity and subcellular localization of Src kinases. Mol Biol Cell 10: 3489-3505, 1999.

25. Amin MA, Matsunaga S, Ma N, et al: Fibrillarin, a nucleolar protein, is required for normal nuclear morphology and cellular growth in HeLa cells. Biochem Biophys Res Commun 360: 320-326, 2007

26. Yanagida M, Hayano T, Yamauchi Y, et al: Human fibrillarin forms a sub-complex with splicing factor 2-associated $\mathrm{p} 32$, protein arginine methyltransferases, and tubulins alpha 3 and beta 1 that is independent of its association with preribosomal ribonucleoprotein complexes. J Biol Chem 279: 1607-1614, 2004
27. Barygina VV, Veiko VP and Zatsepina OV: Analysis of nucleolar protein fibrillarin mobility and functional state in living $\mathrm{HeLa}$ cells. Biochemistry (Mosc) 75: 979-988, 2010.

28. Rajan P, Gaughan L, Dalgliesh C, et al: Regulation of gene expression by the RNA-binding protein Sam68 in cancer. Biochem Soc Trans 36: 505-507, 2008.

29. Bielli P, Busa R, Paronetto MP and Sette C: The RNA-binding protein Sam68 is a multifunctional player in human cancer. Endocr Relat Cancer 18: R91-R102, 2011.

30. Najib S, Martin-Romero C, Gonzalez-Yanes C and SanchezMargalet V: Role of Sam68 as an adaptor protein in signal transduction. Cell Mol Life Sci 62: 36-43, 2005.

31. Sette C: Post-translational regulation of star proteins and effects on their biological functions. Adv Exp Med Biol 693: 54-66, 2010.

32. Fuller SJ, Gillespie-Brown J and Sugden PH: Oncogenic src, raf, and ras stimulate a hypertrophic pattern of gene expression and increase cell size in neonatal rat ventricular myocytes. J Biol Chem 273: 18146-18152, 1998. 